\title{
Classical stabilities of multiplicative inverse difference and adjoint functional equations
}

\section{B.V. Senthil Kumar ${ }^{1 *}$ (D) Khalifa Al-Shaqsi ${ }^{1}$ and Hemen Dutta ${ }^{2}$}

\section{"Correspondence:}

senthilkumar@nct.edu.om

'Department of Information

Technology, Nizwa College of

Technology, Nizwa, Oman

Full list of author information is

available at the end of the article

\section{Springer}

\section{Abstract}

The aim of this present article is to investigate various classical stability results of the multiplicative inverse difference and adjoint functional equations

$$
m_{d}\left(\frac{r s}{r+s}\right)-m_{d}\left(\frac{2 r s}{r+s}\right)=\frac{1}{2}\left[m_{d}(r)+m_{d}(s)\right]
$$

and

$$
m_{a}\left(\frac{r s}{r+s}\right)+m_{a}\left(\frac{2 r s}{r+s}\right)=\frac{3}{2}\left[m_{a}(r)+m_{a}(s)\right]
$$

in the framework of non-zero real numbers. A proper counter-example is illustrated to prove the failure of the stability results for control cases. The relevance of these functional equations in optics is also discussed.

MSC: 39B82; 39B72

Keywords: Multiplicative inverse function; Reciprocal functional equation; Ulam-Hyers stability; Real numbers

\section{Introduction}

The investigation of an approximate solution near to the exact solution of many mathematical equations such as functional, differential, difference and algebraic equations is a fascinating concept in the research domain of pure and applied mathematics. This theory emanated from an inquisitive query proposed in [24] and was developed through different versions, namely Ulam-Hyers Stability (UHS), Ulam-Hyers-T. Rassias Stability (UHTRS), Ulam-Hyers-J. Rassias Stability (UHJRS) and Ulam-Hyers-Rassias-Gavruta Stability (HURGS) by many mathematicians [1, 9, 10, 13, 14]. Recently, this theory has got a lot of momentum in dealing with different types of rational and multiplicative inverse functional equations with their applications in diversified fields, such as physics, electrical engineering, digital image processing. There are more instigating and novel results concerning this topic. For more information, refer to [2-7, 11, 12, 15, 18-22].

(c) The Author(s) 2020. This article is licensed under a Creative Commons Attribution 4.0 International License, which permits use, sharing, adaptation, distribution and reproduction in any medium or format, as long as you give appropriate credit to the original author(s) and the source, provide a link to the Creative Commons licence, and indicate if changes were made. The images or other third party material in this article are included in the article's Creative Commons licence, unless indicated otherwise in a credit line to the material. If material is not included in the article's Creative Commons licence and your intended use is not permitted by statutory regulation or exceeds the permitted use, you will need to obtain permission directly from the copyright holder. To view a copy of this licence, visit http://creativecommons.org/licenses/by/4.0/. 
The rational form of the reciprocal, reciprocal difference and reciprocal adjoint functional equations

$$
\begin{aligned}
& m(r+s)=\frac{m(r) m(s)}{m(r)+m(s)}, \\
& m\left(\frac{r+s}{2}\right)-m(r+s)=\frac{m(r) m(s)}{m(r)+m(s)},
\end{aligned}
$$

and

$$
m\left(\frac{r+s}{2}\right)+m(r+s)=\frac{3 m(r) m(s)}{m(r)+m(s)},
$$

were introduced and obtained their classical stability results in [16, 17].

The multiplicative inverse functional equation

$$
m\left(\frac{r s}{r+s}\right)=m(r)+m(s)
$$

was dealt with in [23] to prove its non-Archimedean stabilities. For the first time in stability theory, a functional equation involving function of a rational argument is considered. It is easy to examine that the reciprocal mapping $m(r)=\frac{1}{r}$ is a solution of (1.4). Induced by the solution of (1.4) and since the power of a thin lens is the multiplicative inverse of its focal length, we focus on the following multiplicative inverse difference and adjoint functional equations, respectively, to investigate their solutions and analytical stabilities:

$$
m_{d}\left(\frac{r s}{r+s}\right)-m_{d}\left(\frac{2 r s}{r+s}\right)=\frac{1}{2}\left[m_{d}(r)+m_{d}(s)\right]
$$

and

$$
m_{a}\left(\frac{r s}{r+s}\right)+m_{a}\left(\frac{2 r s}{r+s}\right)=\frac{3}{2}\left[m_{a}(r)+m_{a}(s)\right],
$$

where $m_{d}, m_{a}: \mathbb{R}^{\star} \longrightarrow \mathbb{R}$ are mappings. We solve Eqs. (1.5) and (1.6). Then we show that there exists an approximate solution near the exact solution of these equations via the Ulam stability idea by considering different upper bounds. We present a suitable example to prove the failure of stability results for singular cases. We also discuss the situations in optics where Eqs. (1.5) and (1.6) could be utilized.

To avoid a singularity in the main results, throughout this paper, let us assume that $\frac{r s}{r+s} \neq$ 0 for all $r, s \in \mathbb{R}^{\star}$.

\section{Equivalency of Eqs. (1.5) and (1.6)}

In the ensuing result, we will prove that Eqs. (1.5) and (1.6) are equivalent and we obtain their solutions.

Theorem 2.1 Assume a mapping $m: \mathbb{R}^{\star} \longrightarrow \mathbb{R}$. Then the following statements are equivalent.

(a) m satisfies (1.4). 
(b) m satisfies (1.5).

(c) m satisfies (1.6).

Hence, the solution of Eqs. (1.5) and (1.6) is a multiplicative inverse mapping.

Proof Initially, assume that $m$ satisfies (1.4). Now, reinstating $(r, s)$ by $(2 r, 2 r)$ in $(1.4)$ and then dividing by 2 , one finds that

$$
m(2 r)=\frac{1}{2} m(r)
$$

for all $r \in \mathbb{R}^{\star}$. Now, replacing $(r, s)$ by $(2 r, 2 s)$ in (1.4) and in lieu of $(2.1)$ in the resultant, one obtains

$$
m\left(\frac{2 r s}{r+s}\right)=\frac{1}{2}[m(r)+m(s)]
$$

for all $r, s \in \mathbb{R}^{\star}$. Now, subtracting (2.2) from (1.4), one arrives at (1.5). Next, let us assume that $m$ satisfies (1.5). If $(r, s)$ is replaced by $(2 r, 2 r)$ in (1.5), then the resultant produces (1.6). Finally, let $m$ satisfy (1.6). By a similar argument as above, substituting $(2 r, 2 r)$ in place of $(r, s)$ in (1.6), and then employing the resulting outcome leads to (1.4).

\section{Stabilities of Eqs. (1.5) and (1.6)}

In this fragment, we investigate various analytical stabilities associated with (1.5) and (1.6) in the domain of non-zero real numbers. To obtain the main results in a simple approach, consider the difference operators $\Delta_{m_{d}}, \Delta_{m_{a}}: \mathbb{R}^{\star} \times \mathbb{R}^{\star} \longrightarrow \mathbb{R}$, defined respectively by

$$
\begin{aligned}
& \Delta_{m_{d}}(r, s)=m_{d}\left(\frac{r s}{r+s}\right)-m_{d}\left(\frac{2 r s}{r+s}\right)-\frac{1}{2}\left[m_{d}(r)+m_{d}(s)\right], \\
& \Delta_{m_{a}}(r, s)=m_{a}\left(\frac{r s}{r+s}\right)+m_{a}\left(\frac{2 r s}{r+s}\right)-\frac{3}{2}\left[m_{a}(r)+m_{a}(s)\right],
\end{aligned}
$$

for all $r, s \in \mathbb{R}^{\star}$.

Theorem 3.1 Assume a fixed constant $\mu= \pm 1$ and a mapping $m_{d}: \mathbb{R}^{\star} \rightarrow \mathbb{R}$. Suppose $\zeta: \mathbb{R}^{\star} \times \mathbb{R}^{\star} \longrightarrow[0, \infty)$ is a function satisfying

$$
\left|\Delta_{m_{d}}(r, s)\right| \leq \zeta(r, s)
$$

for all $r, s \in \mathbb{R}^{\star}$, where $\zeta$ satisfies

$$
\sum_{p=0}^{\infty} \frac{1}{2^{\mu p}} \zeta\left(\frac{r}{2^{\mu(p+1)}}, \frac{s}{2^{\mu(p+1)}}\right)<+\infty
$$

for all $r, s \in \mathbb{R}^{\star}$. Then a unique multiplicative inverse mapping $M_{d}: \mathbb{R}^{\star} \longrightarrow \mathbb{R}$ exists and satisfies (1.5) with the result that

$$
\left|m_{d}(r)-M_{d}(r)\right| \leq \sum_{p=0}^{\infty} \frac{1}{2^{\mu p}} \zeta\left(\frac{r}{2^{\mu(p+1)}}, \frac{r}{2^{\mu(p+1)}}\right)
$$

for all $r \in \mathbb{R}^{\star}$. 
Proof Firstly, let us prove it for the case $\mu=1$. Switching $(r, s)$ to $(r, r)$ in (3.1), we obtain

$$
\left|\frac{1}{2^{\mu}} m_{d}\left(\frac{r}{2^{\mu}}\right)-m_{d}(r)\right| \leq|2|^{\frac{|\mu-1|}{2}} \zeta\left(\frac{r}{2^{\frac{\mu+1}{2}}}, \frac{r}{2^{\frac{\mu+1}{2}}}\right)
$$

for all $r \in \mathbb{R}^{\star}$. Now, replacing $r$ with $\frac{r}{2^{\ell \mu}}$ in (3.4) and then multiplying by $\left|\frac{1}{2}\right|^{\ell \mu}$, we get

$$
\left|\frac{1}{2^{\ell \mu}} m_{d}\left(\frac{r}{2^{\ell \mu}}\right)-\frac{1}{2^{(\ell+1) \mu}} m_{d}\left(\frac{r}{2^{(\ell+1) \mu}}\right)\right| \leq \frac{1}{2^{\ell \mu}} \zeta\left(\frac{r}{2^{(\ell+1) \mu}}, \frac{r}{2^{(\ell+1) \mu}}\right) .
$$

Letting $\ell \rightarrow \infty$ in (3.5) and using (3.2), we see that $\left\{\frac{1}{2^{\ell \mu}} m_{d}\left(\frac{r}{2^{\ell+1) \mu}}\right)\right\}$ is a Cauchy sequence. In lieu of completeness of $\mathbb{R}$, the sequence $\left\{\frac{1}{2^{\ell \mu}} m_{d}\left(\frac{r}{2^{(\ell+1) \mu}}\right)\right\}$ converges to a mapping $M_{d}$ defined by

$$
M_{d}(r)=\lim _{n \rightarrow \infty} \frac{1}{2^{\ell \mu}} m_{d}\left(2^{(\ell+1) \mu} r\right) .
$$

Now, we claim that $M_{d}$ satisfies (1.5). Plugging $(r, s)$ into $\left(\frac{r}{2^{\ell \mu}}, \frac{s}{2^{\ell \mu}}\right)$ in (3.1) and then in the resultant, dividing by $2^{\ell \mu}$ on its both sides, we obtain

$$
\left|\frac{1}{2^{\ell \mu}} \Delta_{m_{d}}\left(\frac{r}{2^{\ell \mu}}, \frac{s}{2^{\ell \mu}}\right)\right| \leq \frac{1}{2^{\ell \mu}} \zeta\left(\frac{r}{2^{\ell \mu}}, \frac{s}{2^{\ell \mu}}\right)
$$

for all $r, s \in \mathbb{R}^{\star}$ and for all positive integers $\ell$. Now, using (3.2), (3.6) in (3.7), we find that $M_{d}$ satisfies (1.5) for all $r, s \in \mathbb{R}^{\star}$. For each $r \in \mathbb{R}^{\star}$ and each integer $\ell$, we have

$$
\begin{aligned}
\left|\frac{1}{2^{\ell \mu}} m_{d}\left(\frac{r}{2^{\ell \mu}}\right)-m_{d}(r)\right| & \leq \sum_{k=0}^{\ell-1}\left|\frac{1}{2^{k \mu}} m_{d}\left(\frac{r}{2^{(k+1) \mu}}\right)-\frac{1}{2^{(k-1) \mu}} m_{d}\left(\frac{r}{2^{k \mu}}\right)\right| \\
& \leq \sum_{k=0}^{\ell-1} \frac{1}{2^{k \mu}} \zeta\left(\frac{r}{2^{(k+1) \mu}}, \frac{r}{2^{(k+1) \mu}}\right) .
\end{aligned}
$$

Applying (3.6) and letting $\ell \rightarrow \infty$, we obtain (3.3). Next we prove that $M_{d}$ is unique. For this, let us consider $M_{d}^{\prime}: \mathbb{R}^{\star} \rightarrow \mathbb{R}$ to be an alternative multiplicative inverse mapping satisfying (1.5) and (3.3). Therefore

$$
M_{d}^{\prime}\left(\frac{r}{2^{\ell \mu}}\right)=2^{\ell \mu} M_{d}^{\prime}(r) \quad \text { and } \quad M_{d}\left(\frac{r}{2^{\ell \mu}}\right)=2^{\ell \mu} M_{d}(r)
$$

for all $r \in \mathbb{R}^{\star}$. By the application of (3.3), we obtain

$$
\begin{aligned}
\left|M_{d}^{\prime}(r)-M_{d}(r)\right| & =2^{-\ell \mu}\left|M_{d}^{\prime}\left(2^{-\ell \mu} r\right)-m_{d}\left(2^{-\ell \mu} r\right)\right| \\
& \leq 2^{-\ell \mu}\left|m_{d}^{\prime}\left(2^{-\ell \mu} r\right)-m_{d}\left(2^{-\ell \mu} r\right)\right|+2^{-\ell \mu}\left|m_{d}\left(2^{-\ell \mu} r\right)-m_{d}\left(2^{-\ell \mu} r\right)\right| \\
& \leq 2 \sum_{p=0}^{\infty} \frac{1}{2^{(\ell+p) \mu}} \zeta\left(\frac{r}{2^{(\ell+p+1) \mu}}, \frac{r}{2^{(\ell+p+1) \mu}}\right) \\
& \leq 2 \sum_{p=\ell}^{\infty} \frac{1}{2^{p \mu}} \zeta\left(\frac{r}{2^{(p+1) \mu}}, \frac{r}{2^{(p+1) \mu}}\right)
\end{aligned}
$$


for all $r \in \mathbb{R}^{\star}$. It can be noticed that $M_{d}$ is unique by allowing $\ell$ to $\infty$ in (3.8). This completes the proof for $\mu=1$. The proof for the case $\mu=-1$ is similar to the above arguments.

The subsequent results are various stabilities pertinent to HUS, HUTRS and HUJRS of Eq. (1.5). The proofs directly follow from Theorem 3.1 for $\mu=-1$. Hence, we omit the proofs. In the ensuing results, let $m_{d}: \mathbb{R}^{\star} \longrightarrow \mathbb{R}$ be a mapping.

Corollary 3.2 Suppose there exists a constant $\eta \geq 0$ (independent of $r$ and s) such that the inequality

$$
\left|\Delta_{m_{d}}(r, s)\right| \leq \frac{\eta}{2}
$$

holds for all $r, s \in \mathbb{R}^{\star}$. Then the limit

$$
M_{d}(r)=\lim _{\ell \rightarrow \infty} \frac{1}{2^{\ell \mu}} m_{d}\left(\frac{r}{2^{\ell \mu}}\right)
$$

exists for all $r \in \mathbb{R}^{\star}, \ell \in \mathbb{N}$ and $M_{d}: \mathbb{R}^{\star} \longrightarrow \mathbb{R}$ is the unique multiplicative inverse mapping exists and satisfies (1.5) with the result that

$$
\left|m_{d}(r)-M_{d}(r)\right| \leq \eta
$$

for all $r \in \mathbb{R}^{\star}$.

Corollary 3.3 For any fixed $\eta_{1} \geq 0$ and $\theta \neq-1$, let the mapping $m_{d}$ satisfy

$$
\left|\Delta_{m_{d}}(r, s)\right| \leq \eta_{1}\left(|r|^{\theta}+|s|^{\theta}\right)
$$

for all $r, s \in \mathbb{R}^{\star}$. Then a unique multiplicative inverse mapping $M_{d}: \mathbb{R}^{\star} \longrightarrow \mathbb{R}$ exists and satisfies (1.5) with the result that

$$
\left|m_{d}(r)-M_{d}(r)\right| \leq \frac{2^{\mu+1} \eta_{1}}{\left|2^{\mu(\theta+1)}-1\right|}|r|^{\theta} \quad \text { for } \theta \neq-1
$$

for all $r \in \mathbb{R}^{\star}$.

Corollary 3.4 Suppose the constants $\gamma$, $\delta$ exist such that $\theta=\gamma+\delta \neq-1$ and $\eta_{2} \geq 0$. Assume the mapping $m_{d}$ satisfies

$$
\left|\Delta_{m_{d}}(r, s)\right| \leq \eta_{2}|r|^{\gamma}|s|^{\delta}
$$

for all $r, s \in \mathbb{R}^{\star}$. Then there exists a unique multiplicative inverse mapping $M_{d}: \mathbb{R}^{\star} \longrightarrow \mathbb{R}$ satisfying (1.5) with the result that

$$
\left|m_{d}(r)-M_{d}(r)\right| \leq \frac{2^{\mu} \eta_{2}}{\left|2^{\mu(\theta+1)}-1\right|}|r|^{\theta} \quad \text { for } \theta \neq-1
$$

and satisfies (1.5) for all $r, s \in \mathbb{R}^{\star}$. 
Inspired by the counter-example in [8], we prove that Eq. (1.5) fails to be stable for $\theta=-1$ in Corollary 3.3 in the domain of non-zero real numbers. For this, the function defined below is used to present a suitable counter-example.

Let a function $v: \mathbb{R}^{*} \longrightarrow \mathbb{R}$ be defined as

$$
v(r)= \begin{cases}\frac{\alpha}{r} & \text { for } r \in(1, \infty), \\ \alpha & \text { elsewhere. }\end{cases}
$$

Let $m_{d}: \mathbb{R}^{*} \longrightarrow \mathbb{R}$ be defined by

$$
m_{d}(r)=\sum_{k=0}^{\infty} 2^{-k} v\left(2^{-k} r\right)
$$

for all $r \in \mathbb{R}^{\star}$. Then the function $m_{d}$ turns into a suitable example to illustrate that (1.5) is unstable for $\theta=-1$ in Corollary 3.3 in the subsequent theorem.

Theorem 3.5 Assume that the function $m_{d}: \mathbb{R}^{*} \rightarrow \mathbb{R}$ described in (3.9) satisfies the inequality

$$
\left|\Delta_{m_{d}}(r, s)\right| \leq 12 \alpha\left(|r|^{-1}+|s|^{-1}\right)
$$

for all $r, s \in \mathbb{R}^{\star}$. Then a multiplicative inverse mapping $M_{d}: \mathbb{R}^{*} \longrightarrow \mathbb{R}$ and a constant $K>0$ do not exist such that

$$
\left|m_{d}(r)-M_{d}(r)\right| \leq K|r|^{-1}
$$

for all $r \in \mathbb{R}^{*}$.

Proof Firstly, let us prove that the mapping $m_{d}$ satisfies (3.11). Using the definition of $m_{d}$, we find $\left|m_{d}(r)\right|=\left|\sum_{k=0}^{\infty} 2^{-k} v\left(2^{-k} r\right)\right| \leq \sum_{k=0}^{\infty} \frac{\alpha}{2^{k}}=2 \alpha$, which indicates that $m_{d}$ is bounded by $2 \alpha$ on $\mathbb{R}$. If $|r|^{-1}+|s|^{-1} \geq 1$, then the expression on the left hand side of (3.11) is less than $12 \alpha$. On the other hand, assume that $0<|r|^{-1}+|s|^{-1}<1$. Hence, there exists an integer $j>0$ such that

$$
\frac{1}{2^{j+1}} \leq|r|^{-1}+|s|^{-1}<\frac{1}{2^{j}} .
$$

As a result, Eq. (3.13) leads to $2^{j}\left(|r|^{-1}+|s|^{-1}\right)<1$, or equivalently; $2^{j} r^{-1}<1,2^{j} s^{-1}<1$. So, $\frac{r}{2^{j}}>1, \frac{s}{2^{j}}>1$. From the last inequalities, we observe that $\frac{r}{2^{j-1}}>2>1, \frac{s}{2^{j-1}}>2>1$ and as a consequence, we have

$$
\frac{1}{2^{j-1}}(r)>1, \quad \frac{1}{2^{j-1}}(s)>1, \quad \frac{1}{2^{j-1}}(r s)>1, \quad \frac{1}{2^{j-1}}(r+s)>1 .
$$

Thus, for every $k=0,1,2, \ldots, j-1$, we acquire

$$
\frac{1}{2^{k}}(r)>1, \quad \frac{1}{2^{k}}(s)>1, \quad \frac{1}{2^{k}}(r s)>1, \quad \frac{1}{2^{k}}(r+s)>1,
$$




$$
\frac{1}{2^{k}}\left(\frac{r s}{r+s}\right)>1, \quad \frac{1}{2^{k}}\left(\frac{2 r s}{r+s}\right)>1
$$

and $\Delta_{m_{d}}\left(2^{-k} r, 2^{-k} s\right)=0$ for $k=0,1,2, \ldots, j-1$. By the application of (3.9) and using the definition of the mapping $m_{d}$, we obtain

$$
\begin{aligned}
\left|\Delta_{m_{d}}(r, s)\right| & \leq \sum_{k=j}^{\infty} \frac{\alpha}{2^{k}}+\sum_{k=j}^{\infty} \frac{\alpha}{2^{k}}+\frac{1}{2} \sum_{k=j}^{\infty} \frac{\alpha}{2^{k}}+\frac{1}{2} \sum_{k=j}^{\infty} \frac{\alpha}{2^{k}} \\
& \leq 3 \alpha \sum_{k=j}^{\infty} \frac{1}{2^{k}} \leq \frac{12 \alpha}{2^{k+1}} \leq 12 \alpha\left(|r|^{-1}+|s|^{-1}\right)
\end{aligned}
$$

for all $r, s \in \mathbb{R}^{*}$, which signifies that the inequality (3.11) holds. Now, we claim that Eq. (1.5) fails to be stable for $\theta=-1$ in Corollary 3.3. On the other hand, let us assume that a multiplicative inverse mapping $m_{d}: \mathbb{R}^{*} \longrightarrow \mathbb{R}$ exists and satisfies (3.12). Then we arrive at

$$
\left|m_{d}(r)\right| \leq(K+1)|r|^{-1}
$$

But we can find a positive integer $m$ with $m \alpha>K+1$. If $r \in\left(1,2^{m-1}\right)$ then $2^{-k} r \in(1, \infty)$ for all $k=0,1,2, \ldots, m-1$ and thus



which contradicts (3.14). Hence we conclude that Eq. (1.5) fails to be stable for $\theta=-1$ in Corollary 3.3.

Remark 3.6 We omit the proof of propositions needed for investigating various stabilities of Eq. (1.6), since they are obtained by similar arguments as in the proof of stability results concerning Eq. (1.5).

\section{Occurrences of (1.5) and (1.6)}

In manufacturing optical instruments, we come across compound lenses. Let the focal lengths of two thin lenses be $r$ and $s$. Then the combined focal length $F$ is given by $F=\frac{r s}{r+s}$. But the power $P$ of a lens is the reciprocal of the focal length $F$. Suppose $p_{1}=\frac{1}{r}$ and $p_{2}=\frac{1}{s}$ are powers of the above lenses, then the combined power $P$ of the lenses is given by $P=$ $r+s$. In view of these theories, we can associate Eqs. (1.5) and (1.6) with the combined focal length and power of lenses. A comparative study of Eqs. (1.1)-(1.6) in various situations of optics is presented below.

- Equation (1.4) interprets the combined power of lenses with forcal length $r$ and $s$ to be equal to half of arithmetic mean of powers of individual lenses; whereas Eq. (1.1) shows that the power of a lens of focal length $r+s$ is equal to half of the harmonic mean of focal lengths $r$ and $s$ of individual lenses.

- Equation (1.5) implies that the difference between the combined power of lenses of focal lengths $r$ and $s$ and the combined power of lenses of focal lengths $2 r$ and $2 s$ is equal to the arithmetic mean of powers of individual lenses with focal lengths $r$ and $s$; while Eq. (1.2) indicates the difference between 2 times the power of a lens of focal 
length $r+s$ and the power of a lens of focal length $r+s$ is equal to half of the harmonic mean of the focal lengths $r$ and $s$ of the individual lenses.

- Equation (1.6) indicates that the sum of the combined power of lenses of focal lengths $r$ and $s$ and the combined power of lenses of focal lengths $2 r$ and $2 s$ is equal to 3 times the arithmetic mean of the powers of the individual lenses with focal lengths $r$ and $s$. But Eq. (1.3) shows the sum of 2 times the power of a lens of focal length $r+s$ and the power of a lens of focal length $r+s$ is equal to three-second of the harmonic mean of the focal lengths $r$ and $s$ of the individual lenses.

\section{Conclusion}

In this study, we have proved that Eqs. (1.4), (1.5) and (1.6) are equivalent, which indicates that the multiplicative inverse mapping is a solution of Eqs. (1.5) and (1.6). In this investigation, zero is omitted to avoid singular cases in obtaining the stability results. We conclude that the stability results of the multiplicative inverse difference and adjoint functional equations hold good in the domain of non-zero real numbers. We have illustrated a suitable example that the stability result of (1.5) fails to hold good for a critical case. We have also discussed the occurrence of Eqs. (1.5) and (1.6) arising in various situations where focal lengths and powers of thin lenses in optics are related.

\section{Acknowledgements}

The authors are thankful to the anonymous referees for their significant comments and suggestions to improve the quality of paper.

\section{Funding}

The first two authors are supported by The Research Council, Oman (Under Project proposal ID: BFP/RGP/CBS/18/099).

\section{Availability of data and materials}

Data sharing is not applicable to this article as no datasets were generated or analyzed during the current study.

\section{Competing interests}

The authors declare that they have no competing interests regarding the publication of this paper.

\section{Authors' contributions}

All authors contributed equally to this paper, participating from drafting to checking and rechecking the manuscript. All authors read and approved the final manuscript.

\section{Author details}

'Department of Information Technology, Nizwa College of Technology, Nizwa, Oman. ${ }^{2}$ Department of Mathematics, Gauhati University, Guwahati, India.

\section{Publisher's Note}

Springer Nature remains neutral with regard to jurisdictional claims in published maps and institutional affiliations.

Received: 14 February 2020 Accepted: 3 May 2020 Published online: 13 May 2020

\section{References}

1. Aoki, T.: On the stability of the linear transformation in Banach spaces. J. Math. Soc. Jpn. 2, 64-66 (1950)

2. Arunkumar, M., Karthikeyan, S.: Fuzzy Banach algebra stability of reciprocal quadratic functional equation via fixed point approach. Int. J. Pure Appl. Math. 119(3), 31-39 (2018)

3. Bodaghi, A.: Intuitionistic fuzzy stability of the generalized forms of cubic and quartic functional equations. Int. J. Fuzzy Syst. 30, 2309-2317 (2016)

4. Bodaghi, A., Kim, S.O.: Approximation on the quadratic reciprocal functional equation. J. Funct. Spaces Appl. 2014 Article ID ID532463 (2014)

5. Bodaghi, A., Rassias, J.M., Park, C.: Fundamental stabilities of an alternative quadratic reciprocal functional equation in non-Archimedean fields. Proc. Jangjeon Math. Soc. 18(3), 313-320 (2015)

6. Bodaghi, A., Senthil Kumar, B.V.: Estimation of inexact reciprocal-quintic and reciprocal-sextic functional equations. Mathematica 49(82), 3-14 (2017)

7. Ebadian, A., Zolfaghari, S., Ostadbashi, S., Park, C.: Approximation on the reciprocal functional equation in several variables in matrix non-Archimedean random normed spaces. Adv. Differ. Equ. 2015, 314 (2015) 
8. Gajda, Z:: On the stability of additive mappings. Int. J. Math. Math. Sci. 14, 431-434 (1991)

9. Găvruta, P.: A generalization of the Hyers-Ulam-Rassias stability of approximately additive mappings. J. Math. Anal. Appl. 184, 431-436 (1994)

10. Hyers, D.H.: On the stability of the linear functional equation. Proc. Natl. Acad. Sci. USA 27, 222-224 (1941)

11. Kim, S.O., Senthil Kumar, B.V., Bodaghi, A.: Stability and non-stability of the reciprocal-cubic and reciprocal-quartic functional equations in non-Archimedean fields. Adv. Differ. Equ. 2017, 77 (2017)

12. Pinelas, S., Arunkumar, M., Sathya, E.: Hyers type stability of a radical reciprocal quadratic functional equation originating from 3 dimensional Pythagorean means. Int. J. Math. Appl. 5(4), 45-52 (2017)

13. Rassias, J.M.: On approximately of approximately linear mappings by linear mappings. J. Funct. Anal. 46, 126-130 (1982)

14. Rassias, T.M.: On the stability of the linear mapping in Banach spaces. Proc. Am. Math. Soc. 72, 297-300 (1978)

15. Ravi, K., Rassias, J.M., Senthil Kumar, B.V.: Ulam stability of generalized reciprocal functional equation in several variables. Int. J. Appl. Math. Stat. 19(D10), 1-19 (2010)

16. Ravi, K., Rassias, J.M., Senthil Kumar, B.V.: Ulam stability of reciprocal difference and adjoint functional equations. Aust. J. Math. Anal. Appl. 8(1), Article ID 13 (2011)

17. Ravi, K., Senthil Kumar, B.V.: Ulam-Gavruta-Rassias stability of Rassias reciprocal functional equation. Glob. J. Appl. Math. Math. Sci. 3(1-2), 57-79 (2010)

18. Ravi, K., Thandapani, E., Senthil Kumar, B.V.: Stability of reciprocal type functional equations. Panam. Math. J. 21(1), 59-70 (2011)

19. Senthil Kumar, B.V. Dutta, H.: Non-Archimedean stability of a generalized reciprocal-quadratic functional equation in several variables by direct and fixed point methods. Filomat 32(9), 3199-3209 (2018)

20. Senthil Kumar, B.V., Dutta, H.: Fuzzy stability of a rational functional equation and its relevance to system design. Int. J. Gen. Syst. 48(2), 157-169 (2019)

21. Senthil Kumar, B.V., Dutta, H.: Approximation of multiplicative inverse undecic and duodecic functional equations. Math. Methods Appl. Sci. 42, 1073-1081 (2019)

22. Senthil Kumar, B.V., Kumar, A., Suresh, G.: Functional equations related to spatial filtering in image enhancement. Int. J. Control Theory Appl. 9(28), 555-564 (2016)

23. Senthil Kumar, B.V., Rassias, J.M., Sabarinathan, S.: Stabilities of various multiplicative inverse functional equations. Tbil. Math. J. 12(4), 15-28 (2019)

24. Ulam, S.M.: Problems in Modern Mathematics, Chapter VI. Wiley, New York (1964)

\section{Submit your manuscript to a SpringerOpen ${ }^{\circ}$ journal and benefit from:}

- Convenient online submission

- Rigorous peer review

- Open access: articles freely available online

- High visibility within the field

- Retaining the copyright to your article

Submit your next manuscript at $\boldsymbol{~ s p r i n g e r o p e n . c o m ~}$ 